\title{
Measuring Impact Rebound with Photography
}

\author{
Hartono Sumali ${ }^{1}$ \\ ${ }^{1}$ Sandia National Laboratories ${ }^{\mathrm{a}}$, Component Science and Mechanics Department, MS 1070, PO Box \\ 5800, Albuquerque, NM 87185, USA
}

\begin{abstract}
To study the rebound of a sphere colliding against a flat wall, a test setup was developed where the sphere is suspended with strings as a pendulum, elevated, and gravity-released to impact the wall. The motion of the sphere was recorded with a highspeed camera and traced with an image-processing program. From the speed of the sphere before and after each collision, the coefficient of restitution was computed, and shown to be a function of impact speed as predicted analytically.
\end{abstract}

\section{Introduction}

This paper discusses a method to record the motion of a sphere rebounding from a flat surface. Results from the experiment can be used for comparing or validating theories on impact rebounds. The theories are useful in many applications. When two solid bodies collide to engage, they bounce off each other, breaking the engagement. The rebound is often critical in the performance of mechanisms. The measurement technique presented here is part of an experiment-based research towards understanding rebounds.

The analytical model we investigate here is for impacts where the speeds are much lower than the acoustic speed in the solids, allowing Hertzian contact theory to apply [1]. Schwager and Pöschel [2] combined Hertzian theory with dynamics to develop a model for the coefficient of restitution for colliding spheres. They assume that the sphere materials behave visco-elastically. To apply the model, first denote $Y=$ Young's modulus, $m=$ effective mass, $\gamma=$ Poisson's ratio, $v_{a}=$ the normal component of the speed of approach, $v_{s}=$ normal speed of separation. From the two colliding spheres' radii $R_{1}$ and $R_{2}$, define an effective radius and an effective mass

$$
R=R_{1} R_{2} /\left(R_{1}+R_{2}\right) ; m=m_{1} m_{2} /\left(m_{1}+m_{2}\right) .
$$

Define a scaled speed number

$$
V=\frac{4}{9}\left[\frac{Y \sqrt{R}}{m\left(1-\gamma^{2}\right)}\right]^{2} v_{a}
$$

\footnotetext{
${ }^{\text {a }}$ This work was conducted at Sandia National Laboratories. Sandia is a multi-program laboratory operated under Sandia Corporation, a Lockheed Martin Company, for the United States Department of Energy under Contract DE-AC04-94-AL85000.
}

This is an Open Access article distributed under the terms of the Creative Commons Attribution-Noncommercial License 3.0, which permits unrestricted use, distribution, and reproduction in any noncommercial medium, provided the original work is properly cited. 
The coefficient of restitution is

$$
C_{R}=\frac{v_{s}}{v_{a}}=1-C_{1} A V^{1 / 5}-C_{2} A^{2} V^{2 / 5}-C_{3} A^{3} V^{3 / 5}-\cdots
$$

The constants $C$ are [3]: $C_{1}=1.153449, C_{2}=-0.798267, C_{3}=0.483582$, and $C_{4}=-0.285279$. Four terms are usually more than necessary especially for low speeds as considered here. The parameter $A$ is related to viscous energy loss in the material, and can be obtained from measurement only [1].

\section{Experiment setup}

Figure 1 shows the sphere under study, suspended by two thin strings that extended to about three times the height of the figure. The sphere was manually lifted and gravity-released to impact a heavy flat wall. The sphere moved in a path assumed to be a circular arc in the $x y$ plane in Fig.1. Upon impact, the sphere rebounded. The motion of the sphere was recorded with a high-speed camera looking into the negative $z$ direction. An image-processing program was used to track the $x$ and $y$ positions of a marker on the sphere.

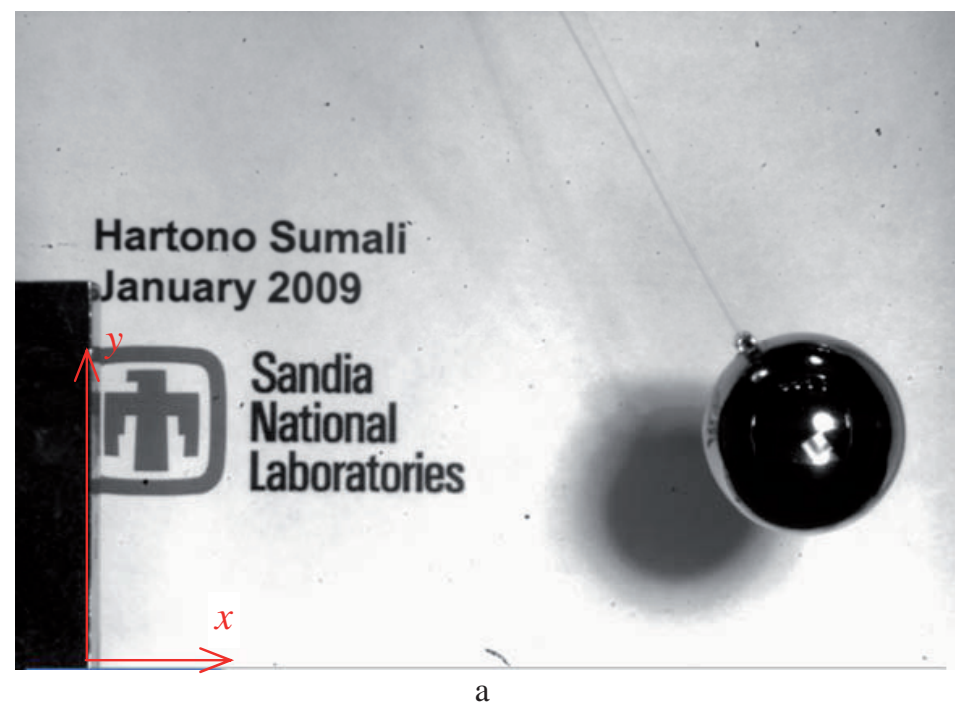

Fig. 1. Sphere suspended by two strings: a. $x y$ plane view; b. $y z$ plane view.

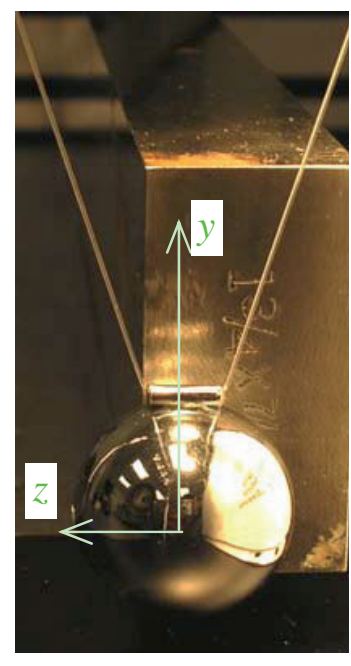

$\mathrm{b}$

\section{Data processing}

To extract data from the images, a circle-fitting routine [4] was used. For measurement points $\left(x_{n}\right.$, $\left.y_{n}\right), N=1, \ldots, N$, scattered near a circular curve, the center of the curve $\left(x_{0}, y_{0}\right)$ and the radius $R$ can be estimated by minimizing the sum of the squared distance between the points and the curve. The least-squares equation to solve for $x_{\mathrm{o}}, y_{\mathrm{o}}$ and $R$ is

$$
\left[\begin{array}{ccc}
\sum_{n=1}^{N} x_{n}{ }^{2} & \sum_{n=1}^{N} x_{n} y_{n} & \sum_{n=1}^{N} x_{n} \\
\sum_{n=1}^{N} x_{n} y_{n} & \sum_{n=1}^{N} y_{n}{ }^{2} & \sum_{n=1}^{N} y_{n} \\
\sum_{n=1}^{N} x_{n} & \sum_{n=1}^{N} y_{n} & N
\end{array}\right]\left\{\begin{array}{c}
-2 x_{0} \\
-2 y_{0} \\
R^{2}-x_{0}{ }^{2}-y_{0}{ }^{2}
\end{array}\right\}=\left\{\begin{array}{c}
-\sum_{n=1}^{N} x_{n}{ }^{3}-\sum_{n=1}^{N} x y_{n}{ }^{2} \\
-\sum_{n=1}^{N} x_{n} y_{n}{ }^{3}-\sum_{n=1}^{N} x_{n}{ }^{2} y_{n} \\
\sum_{n=1}^{N} x_{n}{ }^{2}+\sum_{n=1}^{N} y_{n}{ }^{2}
\end{array}\right\}
$$


The above circle-fit routine was used for computing the scale factor of the images. Eighteen points on the perimeter of the ball in Fig. 1 were picked arbitrarily. The recorded positions of those points were entered into the above routine, which gave the center position and the radius of the ball, all in the unit of pixels. Figure 2 shows the eighteen points and the fit circle. The routine gave $R=$ 85.95 pixels. Separate measurement with a vernier calliper gave $2 R=22.25 \mathrm{~mm}$. Therefore, the images had a scale of $2 * 85.95 / 22.25=7.727$ pixels $/ \mathrm{mm}$.

The above circle-fit routine was also used for identifying the path traced by a marker on the ball in the movie clip. A pattern-tracking program recorded the $x$ - and $y$ positions of the marker in every movie frame. The circle-fit routine gave the numbers and fit path shown in Fig. 3.

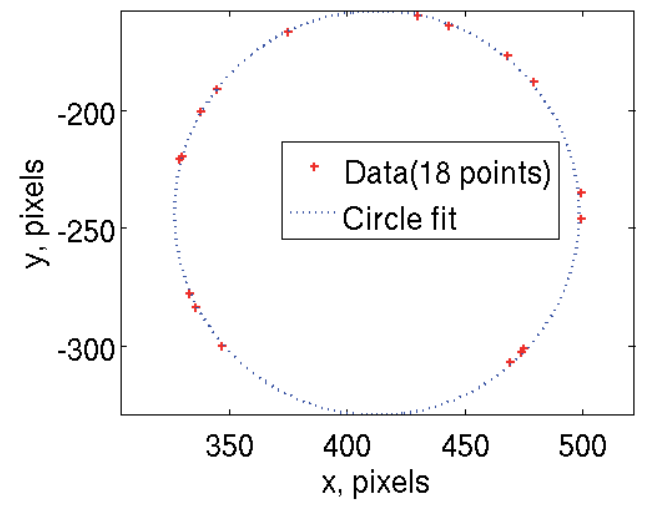

Fig. 2. Circle-fit of ball perimeter.
Fit: $x o=1.161 \mathrm{~mm} ; y_{0}=135.9 \mathrm{~mm} ; R=135.6 \mathrm{~mm}$.

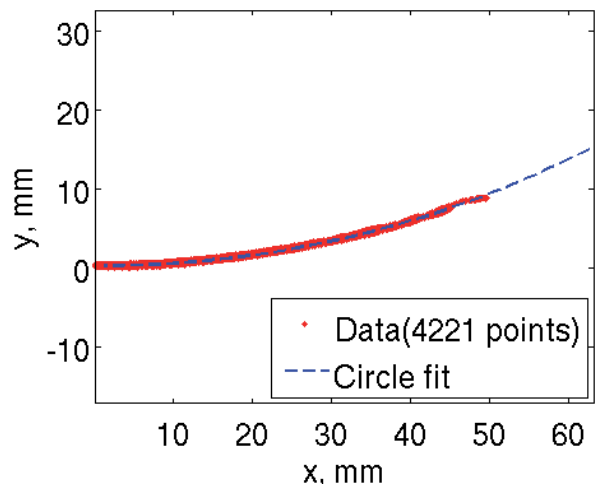

Fig. 3. Circle-fit of ball trajectory.

The $x$ - and $y$ positions of the marker, both from the movie frames and from the circle fit, are plotted against time in Figure 4. The figure shows that the measured displacement in the $x$ direction was much more precise and less noisy than the measured displacement in the $y$ direction, as expected. Therefore, the measured displacement in the $x$ direction was used to compute the displacement in the $y$ direction.

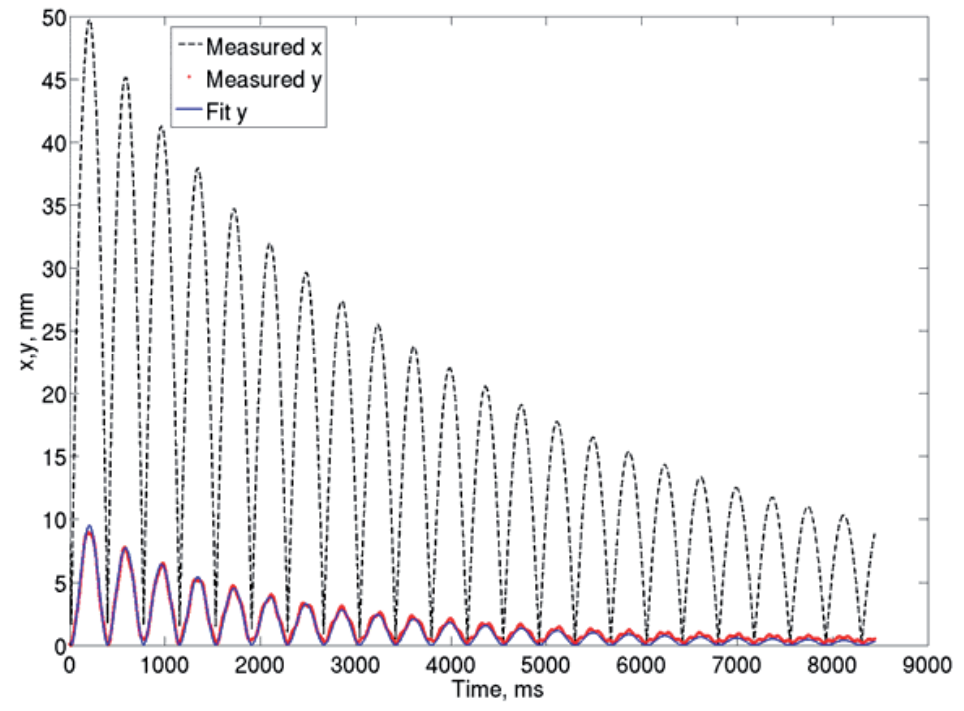

Fig. $4 X$ - and $y$ positions of the sphere versus time. 
The velocities in the $x$ - and $y$ directions were computed as time derivatives. The speed is the magnitude of the velocity vector. Figure 5 shows the speed multiplied by the sign of the $x$ velocity. The dotted curve shows the signed-speed computed using the measured $y$ positions; the solid curve shows the signed-speed computed using the fit $y$ positions. The two curves practically coincide.

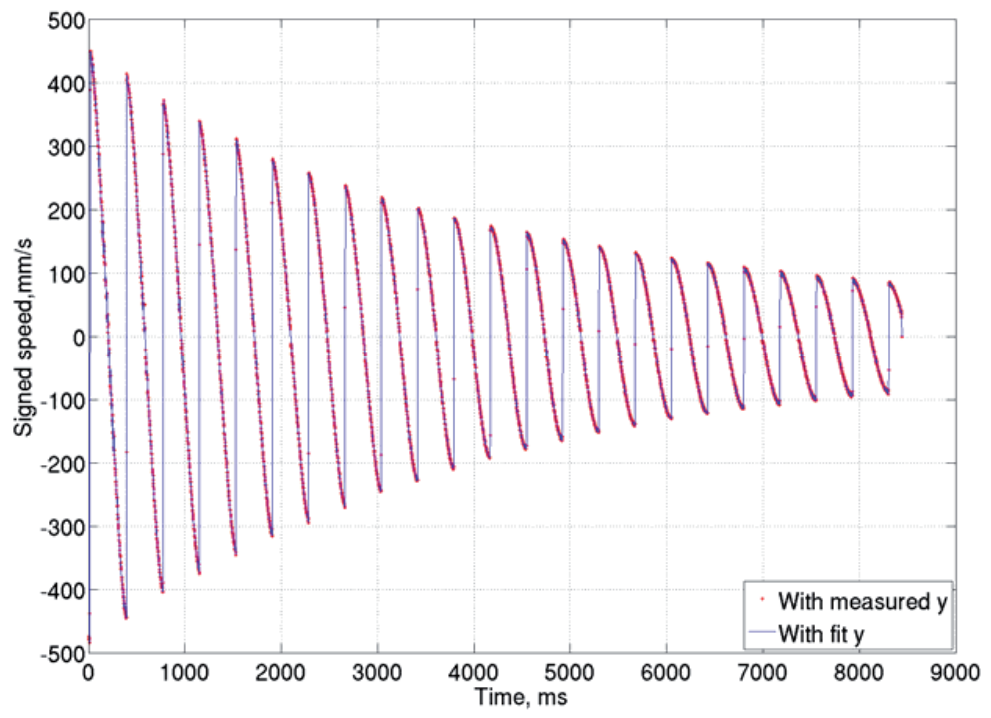

Fig. 5. Signed speed versus time.

The coefficient of restitution was obtained by dividing the speed right after rebound by the speed right before rebound.

\section{Result and remarks}

Figure 6 shows the coefficient of restitution as a function of impact speed that is scaled according to (2). The measured data suggest that the theory predicted correctly the manner in which $\mathrm{CR}$ decreases with impact speed.

\section{References}

[1] N.P. Brilliantov et al., 1996, Phys. Rev. E, 53(5).

[2] T. Schwager and T. Poschel, 1998, Phys. Rev. E, 57(1).

[3] R. Ramirez et al., 1999, Phys. Rev. E, 60(4465). [4] N. Maia et al., 1998, Theoretical and Experimental Modal Analysis, Research Studies Press, England.

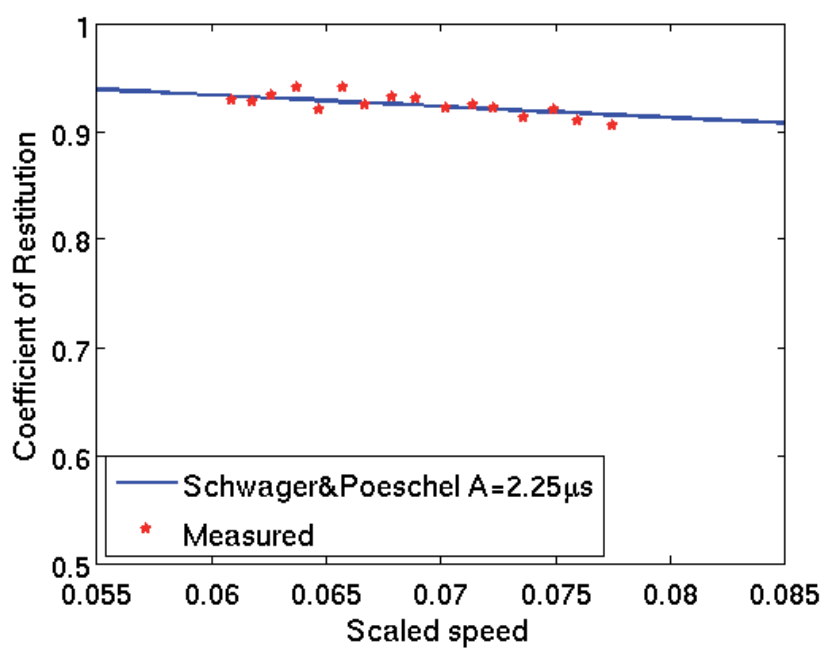

Fig. 6. Coefficient of restitution as a function of nondimensional speed: Theory and measured. 